\title{
Introdução à Supercondutividade, Suas Aplicações e a Mini-Revolução Provocada Pela Redescoberta do $\mathrm{MgB}_{2}$ : Uma Abordagem Didática
}

Introduction to the superconductivity, its applications and the mini-revolution provoked by the rediscovered of the $\mathrm{MgB}_{2}: \mathrm{a}$

didactic approach

Paulo S. Branício

Departamento de Física, Universidade Federal de São Carlos

13565-905, São Carlos, São Paulo

Recebido em 11 de Junho de 2001. Aceito em 29 de Outubro de 2001.

\begin{abstract}
Neste artigo a descoberta da supercondutividade a $39 \mathrm{~K}$ no $\mathrm{MgB}_{2}$ é discutida de forma didática e conceitual. Para salientar sua importância, a história dos supercondutores é descrita brevemente, desde sua descoberta em 1911 no Hg até os dias de hoje. São apontadas as principais características inerentes ao estado supercondutor e indicadas algumas das suas inúmeras aplicações, que estão representadas em ilustrações. O texto apresenta, então, as mudanças que a descoberta do $\mathrm{MgB}_{2}$ traz ao campo de pesquisa e às aplicações. Da maneira como está apresentado, o texto deve ser de interesse não só da comunidade científica em geral, não especializada em supercondutividade, mas em especial dos professores de física do ensino médio, que poderão se valer do texto em atividades envolvendo tópicos de física contemporânea.
\end{abstract}

In this article the discovery of the superconductivity at $39 \mathrm{~K}$ in $\mathrm{MgB}_{2}$ is discussed in a didactic and conceptual way. To point out its importance the history of the superconductors is discribed shortly, from its discovery in 1911 in $\mathrm{Hg}$ up to nowadays. The main inherent characteristics to the superconductor state are pointed out and some of its applications are indicated and represented in illustrations. The text present then the changes that the $\mathrm{MgB}_{2}$ discovery brings to research and applications. In the way it is written, it is believed the text is of interest not only to the general scientific community, not specialized in superconductivity, but especially to high school physics teachers, that could take advantage of the text in activities involving topics of contemporary physics.

\section{Introdução à supercondutivi- dade}

Supercondutores são materiais que perdem a resistência à corrente elétrica quando estão abaixo de uma certa temperatura. A compreensão dos complexos mecanismos que levam alguns materiais a se comportarem como supercondutores vem intrigando os cientistas há quase um século. Tudo começou com o trabalho de Heike Kamerlingh-Onnes[1], que em 1911 descobriu que o $\mathrm{Hg}$ podia transportar corrente elétrica sem nenhuma resistência aparente, como mostrado na Fig. 1. Além disso, esta corrente podia persistir por um tempo indefinido. Onnes conseguiu esse feito trabalhando em seu laboratório de baixas temperaturas em Leiden na Holanda, onde alguns anos antes tinha conseguido liquefazer o hélio pela primeira vez. Ele deu o nome de supercondutividade ao estranho fenômeno. A par- tir de então, o termo supercondutores vem sendo usado para denotar todos os materiais que, abaixo de uma certa temperatura crítica, $\mathrm{T}_{c}$, perdem a resistência à passagem de corrente elétrica, além de apresentar outras propriedades. Dois anos após a descoberta, em 1913, Onnes é agraciado com o prêmio Nobel de Física. Em seu discurso, ele observa ainda que o estado supercondutor podia ser destruído aplicando-se um campo magnético suficientemente grande.

A partir da descoberta inicial, a supercondutividade foi sendo procurada em vários materiais e, em especial, nos metais, que são naturalmente bons condutores de eletricidade. Em 1929, o $\mathrm{T}_{c}$ recorde estava com o Nióbio em $9.25 \mathrm{~K}$; em 1941 com a liga de Nióbio-Nitrogênio em $16 \mathrm{~K}$; já em 1953 com a liga de Vanádio-Silício em 17.5 $\mathrm{K}$; e dai por diante. Até 1986 , o $\mathrm{T}_{c}$ recorde estava com a liga de Nióbio e Germânio em 23.2 K, quando então os supercondutores de alta temperatura foram descobertos. A supercondutividade a baixas temperaturas 
foi

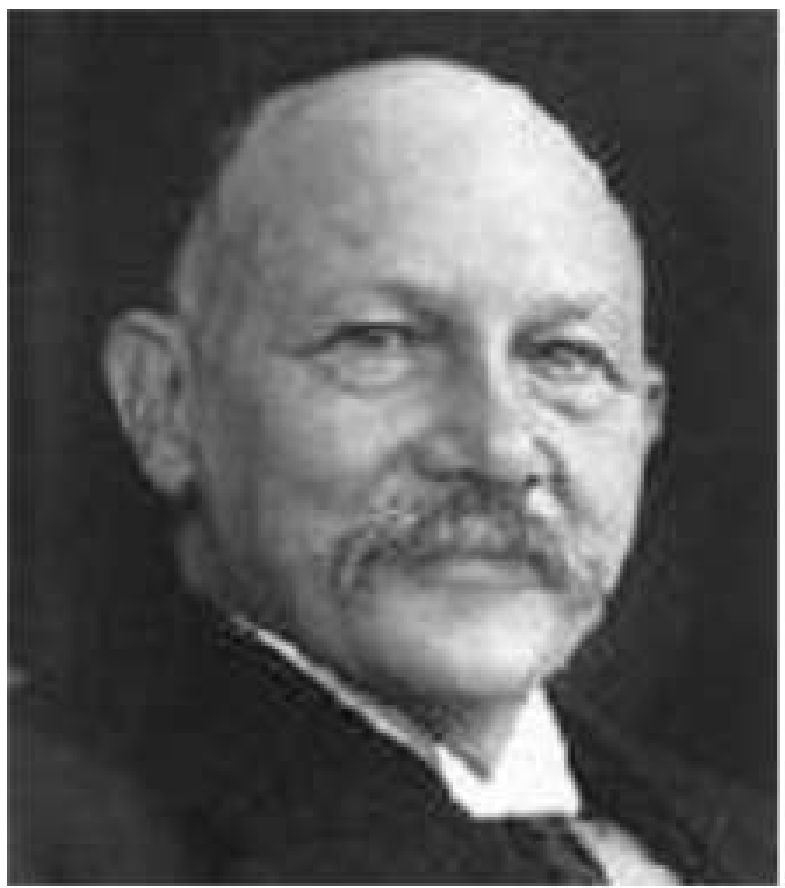

Figura 1. Heike Kamerlingh-Onnes, descobridor da supercondutividade e ganhador do prêmio Nobel de Física de 1913 (figura retirada da página da internet superconductors.org[3]).

descrita em 1957 pela teoria BCS, desenvolvida pelos cientistas americanos John Bardeen, Leon Cooper e John Schrieffer. Em 1972, eles foram agraciados com o prêmio Nobel de Física em reconhecimento à importância desta teoria. No entanto, mesmo sendo precisa na explicação da supercondutividade a baixas temperaturas em metais e ligas simples, a teoria $\mathrm{BCS}$ revelou-se ineficiente na descrição completa da supercondutividade a altas temperaturas, como nas cerâmicas supercondutoras descobertas na década de 80. A primeira delas, $\mathrm{La}_{2-x} \mathrm{Ba}_{x} \mathrm{CuO}_{4}$, foi descoberta por Alex Müller e Georg Bednorz em 1986. Essa descoberta foi de tal importância que mudou notadamente os rumos da supercondutividade. O fato mais marcante foi que a supercondutividade foi descoberta em um material até então considerado isolante, ou seja, que normalmente é um péssimo condutor de eletricidade. Até então, os cientistas não tinham considerado seriamente a possibilidade de um material como este ser um supercondutor, muito menos de ter um $\mathrm{T}_{c}$ maior que o dos metais. No ano seguinte, Müller e Bednorz ganham o prêmio Nobel de Física pela descoberta. De 1987 até hoje, os cientistas passaram a procurar intensamente novas cerâmicas supercondutoras e dezenas delas foram descobertas elevando o valor recorde de $\mathrm{T}_{c}$ a incríveis $138 \mathrm{~K}$ para o composto $\mathrm{Hg}_{0.8} \mathrm{Tl}_{0.2} \mathrm{Ba}_{2} \mathrm{Ca}_{2} \mathrm{Cu}_{3} \mathrm{O}_{8.33}$, a pressão ambiente. Em contrapartida, a procura de novos compostos metálicos supercondutores foi deixada de lado devido ao entusiasmo gerado com as possibilidades abertas com a descoberta dos supercondu- tores de alto $\mathrm{T}_{c}$. Em janeiro de 2001, a comunidade científica é sacudida novamente com a descoberta da supercondutividade no composto metálico $\mathrm{MgB}_{2}$, a 39.2 $\mathrm{K}$. Nada menos que $16 \mathrm{~K}$ maior que qualquer composto metálico até então conhecido. Para melhor apreciar a importância desta descoberta e como ela está revolucionando o estudo da supercondutividade, vamos entender com mais detalhes algumas características dos supercondutores e algumas de suas aplicações.

\section{A. Supercondutores Tipo 1 e Tipo 2}

Os supercondutores são divididos em dois tipos, de acordo com suas propriedades específicas. Os supercondutores do Tipo 1 são formados principalmente pelos metais e por algumas ligas e, em geral, são condutores de eletricidade à temperatura ambiente. Eles possuem um $\mathrm{T}_{c}$ extremamente baixo, que, segundo a teoria BCS, seria necessário para diminuir as vibrações dos átomos do cristal e permitir o fluxo sem dificuldades dos elétrons pelo material, produzindo assim a supercondutividade. Os supercondutores desse tipo foram os primeiros a serem descobertos e os cientistas verificaram que a transição para o estado supercondutor a baixa temperatura tinha características peculiares: ela acontecia abruptamente, veja Fig. 2(a), e era acompanhada pelo efeito Meissner. Esse efeito, que talvez seja a característica mais famosa dos supercondutores, é a causa da levitação magnética de um imã, por exemplo, quando é colocado sobre um pedaço de supercondutor. A explicação para o fenômeno está na repulsão total dos campos magnéticos externos pelos supercondutores do Tipo 1, o que faz com que o campo magnético interno seja nulo, desde que o campo externo aplicado não seja muito intenso. A maioria dos materiais, como vidro, madeira e água, também repele campos magnéticos externos, o que faz com que o campo no interior deles seja diferente do campo externo aplicado. Esse efeito é chamado de diamagnetismo e tem sua origem no movimento orbital dos elétrons ao redor dos átomos, que cria pequenos "loopings" de correntes. Elas, por sua vez, criam campos magnéticos, segundo as leis da eletricidade e magnetismo e, com a aplicação de campo magnético externo tendem a se alinhar de tal forma que se oponham ao campo aplicado. No caso dos condutores, além do alinhamento do movimento orbital dos elétrons, correntes de blindagem são induzidas no material e cancelam parte do campo magnético no seu interior. Se considerarmos um condutor ideal, ou seja, que não apresenta resistência à corrente elétrica, o cancelamento do campo é total, caracterizando o chamado "diamagnetismo perfeito". Nos supercondutores do Tipo 1, o cancelamento do campo magnético interno também é total, porém esse comportamento é distinto do diamagnetismo perfeito. Como podemos ver na Fig. 3 , os supercondutores do Tipo 1, no estado supercondutor, possuem campo magnético nulo no seu interior, 
mesmo no caso de o campo magnético externo ser diferente de zero antes da transição supercondutora, diferente do comportamento de um condutor ideal.
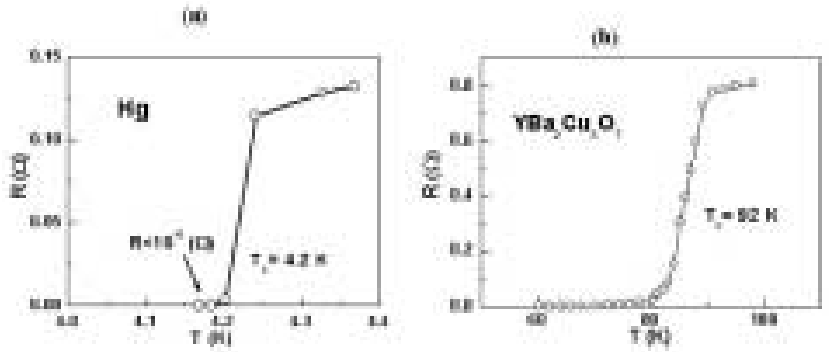

Figura 2. Exemplos de transição supercondutora para supercondutores do Tipo 1 em (a) e Tipo 2 em (b). Em (a) temos a transição do Mercúrio, que foi o primeiro supercondutor descoberto (dados retirados do trabalho original de Onnes[1]). Pode-se ver que a transição para o regime supercondutor é acentuada, ao contrário de (b) no qual uma região intermediária existe (dados retirados do artigo de $\mathrm{Wu}$ et al.[2]).

\section{(a) Diamagneto perfeito}

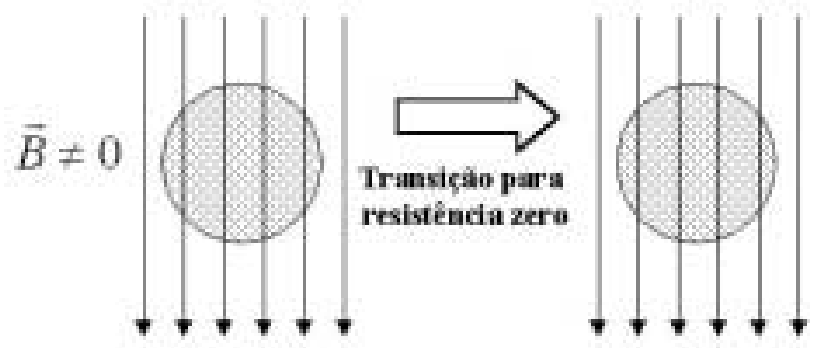

(b) Supercondutor do tipo 1

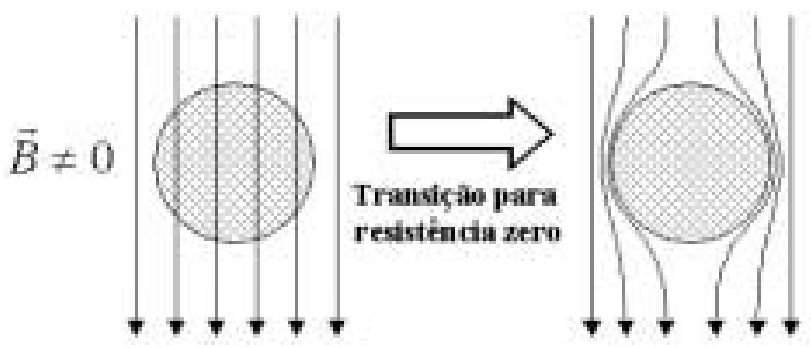

Figura 3. Diferença entre um diamagneto perfeito, em (a), e um supercondutor do Tipo 1, em (b). Se um condutor, em (a), possui campo magnético em seu interior e é, então, resfriado até o estado de resistência zero, transformando-se em um diamagneto perfeito, o campo magnético no seu interior se mantém inalterado. De modo distinto, um supercondutor do Tipo 1, em (b), sempre exclui qualquer campo magnético presente no seu interior no momento em que passa do estado normal para o estado supercondutor.

Praticamente todos os metais são supercondutores do Tipo 1, a temperaturas suficientemente baixas. Entre eles, temos ( $\mathrm{T}_{c}$ em Kelvin entre parênteses): $\mathrm{Pb}$ (7.2), Hg (4.15), Al (1.175), Ti (0.4), U (0.2), W (0.0154), Rh (0.000325). Curiosamente os melhores condutores disponíveis, que são o ouro $(\mathrm{Au})$, a prata $(\mathrm{Ag})$ e o Cobre $(\mathrm{Cu})$ não são supercondutores.

Já os supercondutores do Tipo 2 são formados por ligas metálicas e outros compostos. As exceções são os metais puros, Vanádio (V), Tecnécio (Tc) e Nióbio (Nb). Em geral, as temperaturas críticas associadas a eles são muito mais altas que as dos supercondutores do Tipo 1, como é o caso das cerâmicas baseadas em óxidos de cobre. No entanto, o mecanismo atômico que leva à supercondutividade neste tipo de supercondutor, até hoje não está completamente desvendado. O primeiro material supercondutor do Tipo 2 descoberto foi uma liga de chumbo e bismuto fabricada em 1930 por W. de Haas e J. Voogd. Eles perceberam que a liga apresentava características distintas dos supercondutores convencionais, Tipo 1. A transição para o estado supercondutor era gradual, com a presença de um estado intermediário, como está mostrado na Fig 2(b). Além disso, o efeito Meissner não era perfeito: o material permitia a penetração de algum campo magnético, de modo contrário aos supercondutores do Tipo 1. No estado intermediário, o supercondutor do Tipo 2 apresenta regiões no estado normal, cercada por regiões supercondutoras, como é mostrado na Fig. 4(a). Essas regiões mistas, chamadas de vórtices, permitem a penetração de campo magnético no material, através dos núcleos normais. Conforme a temperatura aumenta, dentro do estado intermediário, os núcleos vão superando as regiões supercondutoras, como é mostrado na Fig. 4(b). Isso acontece até a perda completa do estado supercondutor, quando os núcleos normais se sobrepõem.

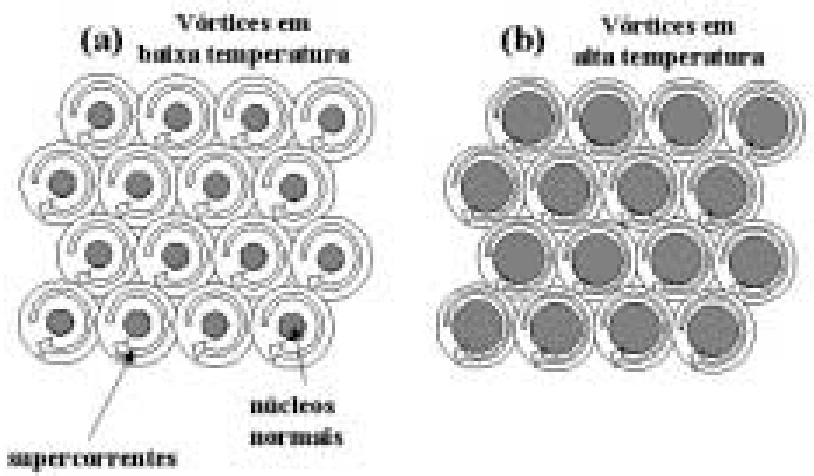

Figura 4. Estrutura de vórtices dos supercondutores do Tipo 2. No estado intermediário, os supercondutores do Tipo 2 apresentam regiões no estado normal cercadas por regiões supercondutoras, como é mostrado em (a). Para temperaturas maiores, dentro da região intermediária, os núcleos normais ficam mais compactados, como é mostrado em (b).

Todos os supercondutores de alta temperatura pertencem ao Tipo 2, incluindo-se o recordista atual, que pertence à classe das cerâmicas baseadas em óxidos de cobre (cupretos). A seguir, enumeramos alguns destes compostos com seu respectivo $T_{c}$ em Kelvin, entre parênteses: 
$\mathrm{Hg}_{0.8} \mathrm{Tl}_{0.2} \mathrm{Ba}_{2} \mathrm{Ca}_{2} \mathrm{Cu}_{3} \mathrm{O}_{8.33} \quad$ (138), $\quad \mathrm{Bi}_{2} \mathrm{Sr}_{2} \mathrm{Ca}_{2} \mathrm{Cu}_{3} \mathrm{O}_{10}$ (115), $\mathrm{Ca}_{1-x} \mathrm{Sr}_{x} \mathrm{CuO}_{2}$ (110), $\mathrm{TmBa}_{2} \mathrm{Cu}_{3} \mathrm{O}_{7}$ (101), $\mathrm{YBa}_{2} \mathrm{Cu}_{3} \mathrm{O}_{7+}$ (93), $\mathrm{La}_{1.85} \mathrm{Ba}_{15} \mathrm{CuO}_{4}$ (35), $\mathrm{CsC}_{60}$ (40), $\mathrm{MgB}_{2}$ (39.2), $\mathrm{Nb}_{3} \mathrm{Ge}$ (23.2) e os metais $\mathrm{Nb}$ (9.25), $\mathrm{Tc}(7.8)$ e $\mathrm{V}(5.4)$. $\overrightarrow{\mathbf{B}}$

\section{B. Teoria BCS}

A teoria que viria a explicar satisfatoriamente a supercondutividade a baixas temperaturas, presente nos supercondutores do Tipo 1, apareceu somente em 1957, graças ao trabalho de John Bardeen, Leon Cooper e Robert Schrieffer. Um ponto chave na teoria criada por eles é a formação de pares de elétrons, conhecidos como pares de Cooper, através de interações com oscilações da rede cristalina. Esta teoria é conhecida hoje como teoria BCS, nome formado com as iniciais dos sobrenomes dos autores, que podem ser vistos na Fig. 5. Os elétrons, assim como todas as partículas com spin fracionário, são chamados de férmions e obedecem ao princípio de exclusão de Pauli, o qual proíbe que duas partículas ocupem o mesmo nível de energia. No entanto, os pares de Cooper se comportam de maneira muito diferente do de elétrons isolados. Eles atuam como bósons, partículas de spin inteiro, podendo se condensar em um mesmo nível de energia. Os pares de Cooper podem ser comparados a duas bolas de boliche nas bordas de um colchão de água, como mostrado na Fig 6. Conforme alguém empurra uma das bolas, o colchão se deforma e a deformação atrai a segunda bola.
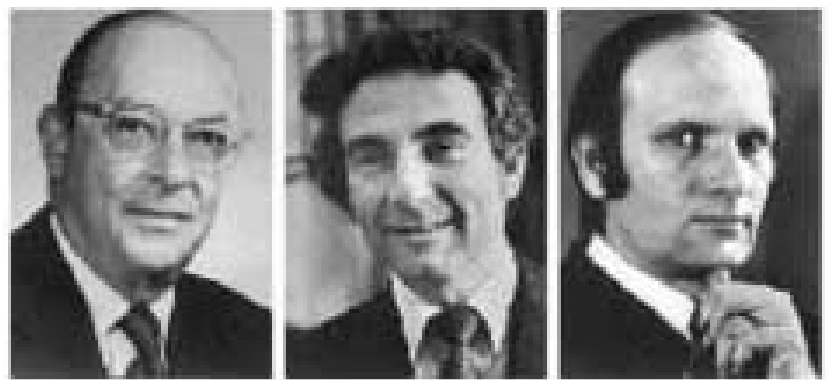

Figura 5. John Bardeen, Leon Cooper e John Schrieffer, criadores da teoria BCS, que descreve a supercondutividade a baixas temperaturas, e ganhadores do prêmio Nobel de Física de 1972 (figura retirada da página da internet superconductors.org[3]).

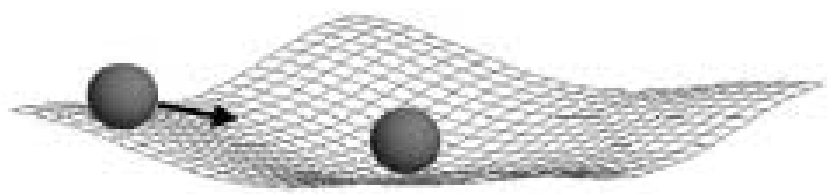

Figura 6. Representação da atração entre elétrons na formação dos pares de Cooper. Assim como uma bola de boliche é atraída por outra que está rolando ao meio de um colchão de água, como pode ser visto na figura, um elétron é atraído pela deformação na rede cristalina provocada por outro elétron com spin e momento contrários (figura adaptada do artigo original de Ostermann et al.[5]).
Em suma, na teoria BCS, a atração entre pares de elétrons mediada por oscilações da rede cristalina é a responsável pela supercondutividade. Os pares de Cooper formam um condensado que flui sem resistência pelo material e atua expelindo campos magnéticos externos fracos, ocasionando o efeito Meissner. Uma discussão mais detalhada da teoria BCS e dos supercondutores do Tipo 1 pode ser encontrada no artigo de Ostermann et al.[5]

\section{Supercondutores a altas temperaturas}

Mesmo tendo muito sucesso na explicação da supercondutividade a baixas temperaturas, a teoria BCS não explica satisfatoriamente o fenômeno a altas temperaturas. O primeiro material dessa classe foi descoberto 15 anos atrás e deixou a comunidade científica perplexa, pois a supercondutividade havia sido descoberta em cerâmicas, um material que geralmente é isolante, e o mais impressionante, em torno de $30 \mathrm{~K}$. Os descobridores, George Bednorz e Alex Müller[6], podem ser vistos na Fig. 7. A descoberta se tornou ainda mais surpreendente quando, em novembro de 1986, Paul Chu da Universidade de Houston e Mang-Kang Wu da Universidade do Alabama, descobriram que o $\mathrm{YBa}_{2} \mathrm{Cu}_{3} \mathrm{O}_{7}$, simbolizado por YBCO e mostrado na Fig. 8, com sua estrutura de camadas, superconduzia a $93 \mathrm{~K}$, ou seja, a temperaturas superiores à temperatura do nitrogênio líquido $(77 \mathrm{~K})$. Ficou claro naquele ano que uma revolução na Física havia começado. No ano seguinte, em uma sessão especial da reunião de março da Sociedade Americana de Física em Nova Iorque, ocorreu a celebração do começo da nova era da supercondutividade. Este evento, que ficou conhecido como o "Woodstock" da Física, reuniu mais de 3000 pessoas na sala de apresentação principal, com outras 3000 pessoas assistindo em circuito de televisão fechado, do lado de fora. Nos anos seguintes, várias outras cerâmicas supercondutoras foram descobertas, todas baseadas em óxidos de cobre, incluindo aquelas com tálio e mercúrio que hoje apresentam as maiores temperaturas críticas.

\section{Aplicações de supercondu- tores}

Os supercondutores são materiais muito interessantes para uso em várias aplicações devido às suas propriedades peculiares. A maioria das suas aplicações se vale da resistividade nula, que em alguns aparelhos elétricos é sinônimo de eficiência máxima, como é o caso dos geradores de eletricidade e dos cabos de transmissão, que não têm perda de energia elétrica por calor. Outras aplicações se valem dos altos campos magnéticos 


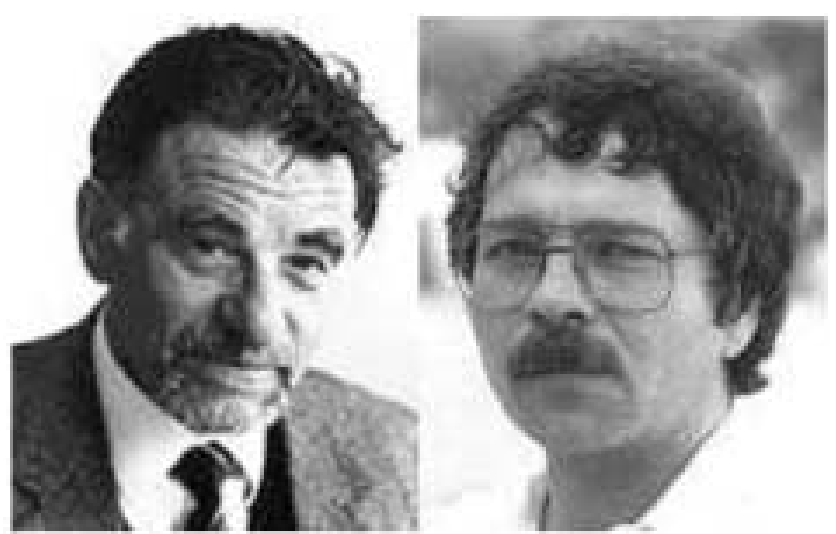

Figura 7. Alex Müller e Georg Bednorz, descobridores das cerâmicas supercondutoras e ganhadores do prêmio Nobel de Física de 1987 (figura retirada da página da internet superconductors.org[3]).

\section{$\mathrm{YBa}_{2} \mathrm{Cu}_{3} \mathrm{O}_{7}$}

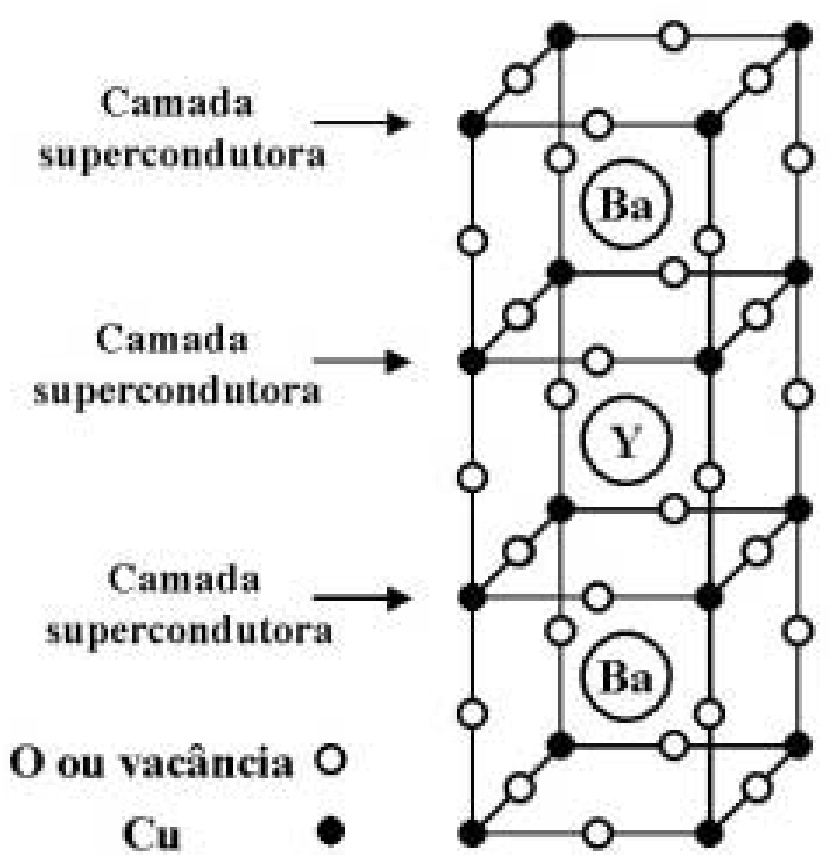

Figura 8. Estrutura perovskita da cerâmica supercondutora $\mathrm{YBa}_{2} \mathrm{Cu}_{3} \mathrm{O}_{7}$, mostrando as camadas supercondutoras de $\mathrm{Cu}$ e $\mathrm{O}$ e o reservatório de carga. (figura adaptada do livro "Superconductivity: its historical roots and development from mercury to the ceramic oxides" de P. F. Dahl[7])

que podem ser obtidos eficientemente com magnetos supercondutores. Os aparelhos de ressonância magnética, por exemplo, assim como os trens flutuantes (Maglev) e alguns aparelhos utilizados no estudo de materias utilizam estes campos. As outras aplicações mais comuns se valem do efeito Meissner.

\section{A. Produção e transmissão de eletrici- dade}

Uma aplicação ideal para os supercondutores seria a transmissão de energia elétrica das estações geradoras para as cidades. Entretanto, isso está longe de ser economicamente viável devido ao alto custo e à dificuldade técnica de se refrigerar vários quilômetros de cabos supercondutores a temperaturas criogênicas, embora cabos de até 45 metros possam ser encontrados em utilização. Cabos de 120 metros, capazes de transportar 100 milhões de watts estão sendo construídos pela empresa americana Pirelli Wire e devem entrar em operação brevemente em uma subestação em Frisbie, Detroit. Na Fig. 9, podemos ver um cabo BSCCO $\left(\mathrm{Bi}_{2} \mathrm{Sr}_{2} \mathrm{CaCu}_{2} \mathrm{O}_{9}\right)$ resfriado com nitrogênio líquido. Já a construção e utilização de geradores de eletricidade em usinas geradoras têm grande potencial. Como a eficiência desses geradores é maior que $99 \%$ e seu tamanho é a metade daquele dos geradores convencionais feitos de cobre, eles são muito atrativos e várias empresas têm planos para construí-los. A empresa americana General Eletric é uma delas e está atualmente desenvolvendo um protótipo capaz de gerar 100 MVA (megawatt-ampere).

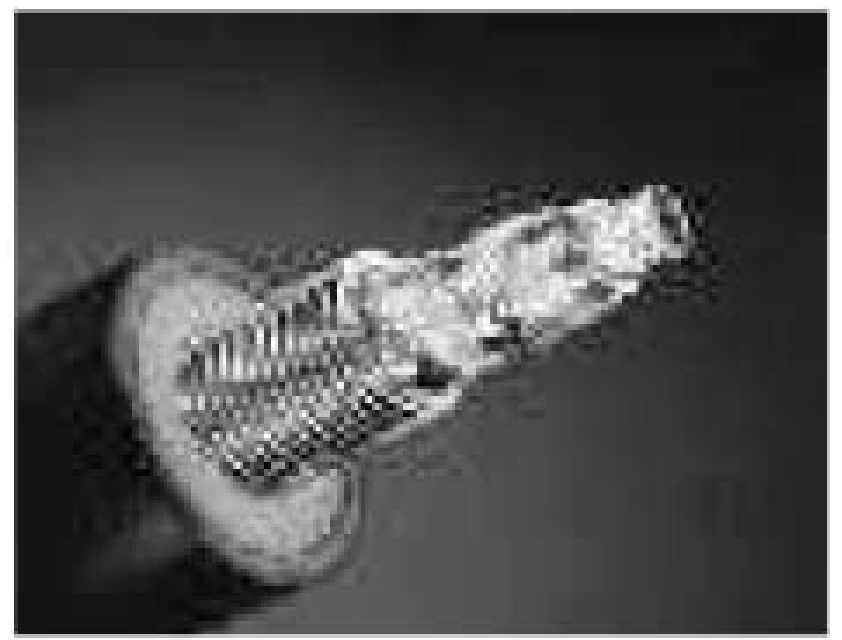

Figura 9. Cabo supercondutor de BSCCO pesando $1 \mathrm{Kg} / \mathrm{m}$ equivalente a um cabo de cobre pesando $72 \mathrm{Kg} / \mathrm{m}$ (figura retirada da página da internet superconductors.org[3]).

Além de produzir e transmitir eletricidade, os supercondutores podem também ser usados para armazenála. Existem dois tipos principais de baterias que podem ser construídas. O primeiro tipo é o das SMES (superconducting magnetic energy storage), veja Fig. 10(a), que podem ser descritas como bobinas gigantes, mantendo uma alta corrente, que podem ser usadas quando desejado. O segundo tipo é chamado comumente de "flywheel" e consiste em um imã permanente em formato cilíndrico, com grande massa, girando com alta 
velocidade sobre um supercondutor, veja Fig. 10(b). Esta bateria utiliza-se do efeito Meissner, que faz os supercondutores repelirem fortemente qualquer imã permanente. (a)

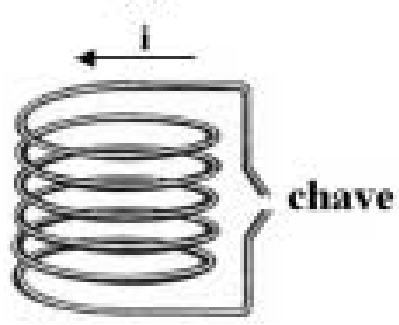

(b)

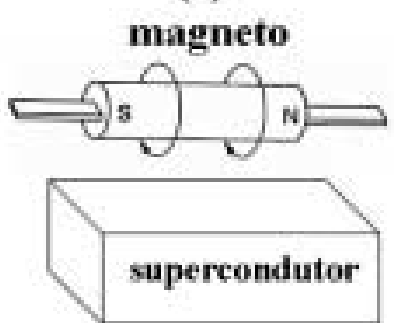

Figura 10. Tipos de baterias supercondutoras. SMES (Superconducting magnetic energy storage) em (a) e Flywheel em (b).

As baterias supercondutoras são especialmente interessantes na estabilização de redes elétricas, em especial as SMES. Em março de 2000, por exemplo, foi encomendada a fabricação de um conjunto delas SMES para a estabilização da rede do estado de Winconsin - EUA. Um conjunto destas SMES é capaz de reservar mais de 3 milhões de watts para ser usado durante pequenos blecautes.

\section{B. Trem magneticamente levitado (MA- GLEV)}

Como altas correntes elétricas podem ser mantidas nos supercondutores, altos campos magnéticos podem ser gerados, de acordo com as leis da eletricidade e magnetismo. Uma das aplicações é a levitação magnética que pode ser utilizada em veículos de transporte, como trens, eliminando a fricção com os trilhos. Trens desse tipo podem ser feitos com magnetos convencionais, pois utilizam basicamente atração e repulsão magnéticas na levitação. Entretanto, os magnetos convencionais desperdiçam energia elétrica na forma de calor e precisam ser bem maiores que os magnetos supercondutores para fornecerem os campos magnéticos necessários à levitação. Na década de 90 , trens comerciais começaram a ser desenvolvidos principalmente no Japão, onde o desenvolvimento da tecnologia MAGLEV ganhou apoio maciço do governo. Recentemente o último protótipo desenvolvido, MLX01 (veja Fig. 11), chegou a $552 \mathrm{Km} / \mathrm{h}$ em uma composição tripulada, de 5 vagões. Outros trens estão sendo desenvolvidos e devem entrar em operação nos próximos anos na Alemanha e nos Estados Unidos.

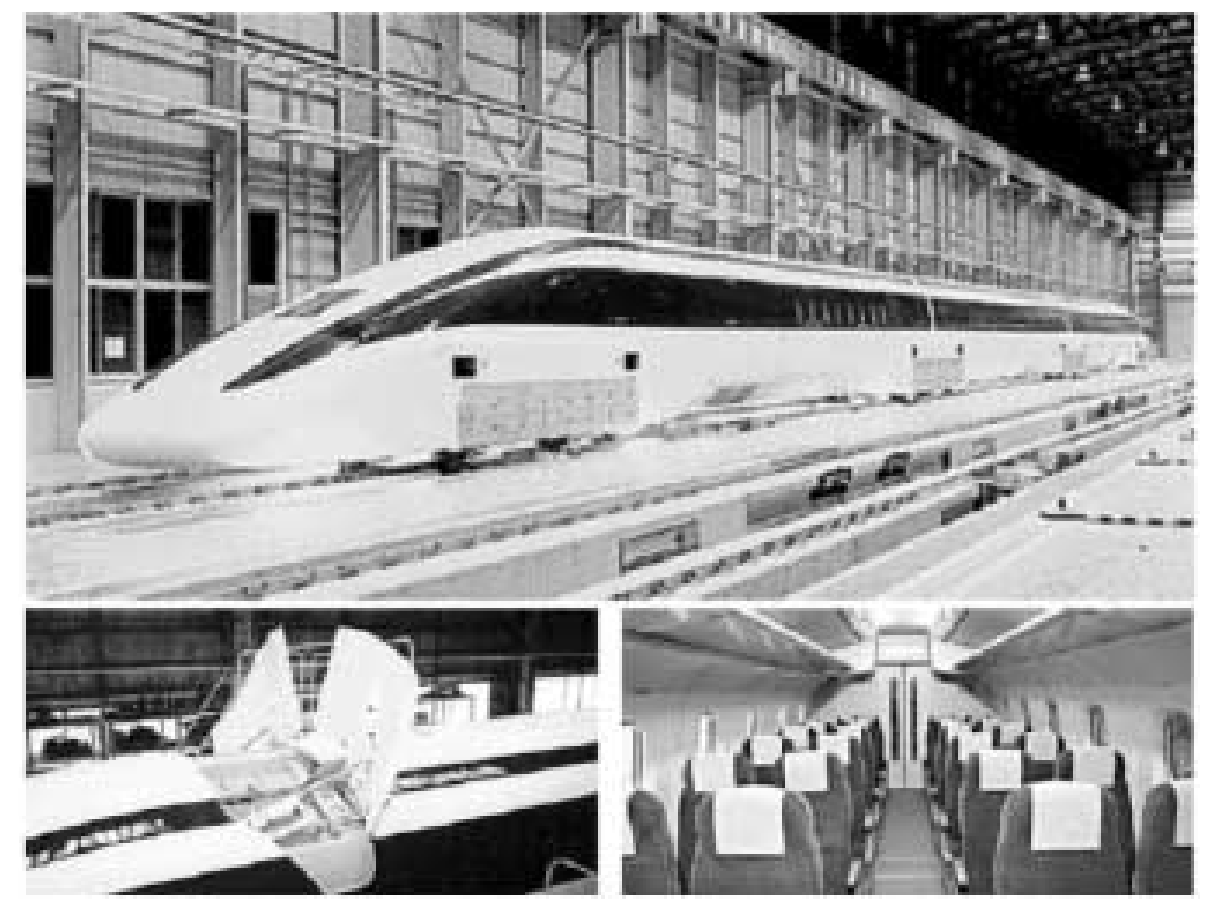

Figura 11. Protótipo de trem magneticamente levitado (MAGLEV) MLX01 construído em 1997. Em detalhe os freios aerodinâmicos e as poltronas (figura retirada da página da internet do projeto MAGLEV[8]).

\section{Ressonância magnética nuclear}

Outra aplicação para os altos campos magnéticos obtidos dos supercondutores é a fabricação e utilização de aparelhos de ressonância magnética nuclear
(RMN). O princípio de funcionamento desses aparelhos é baseado na ressonância que os átomos de hidrogênio entram na aplicação de campo magnético forte. Os átomos de $\mathrm{H}$ presentes nas moléculas de água e de gor- 
dura absorvem a energia magnética e a emitem numa freqüência, que é, então, detectada e analisada graficamente em um computador. A Fig. 12 mostra uma imagem por RMN. O diagnóstico através de imagens deste tipo tornou-se atualmente um procedimento médico indispensável devido, principalmente, ao desenvolvimento da capacidade de processamento dos computadores, necessária na análise da grande quantidade de dados que é gerada durante os exames.

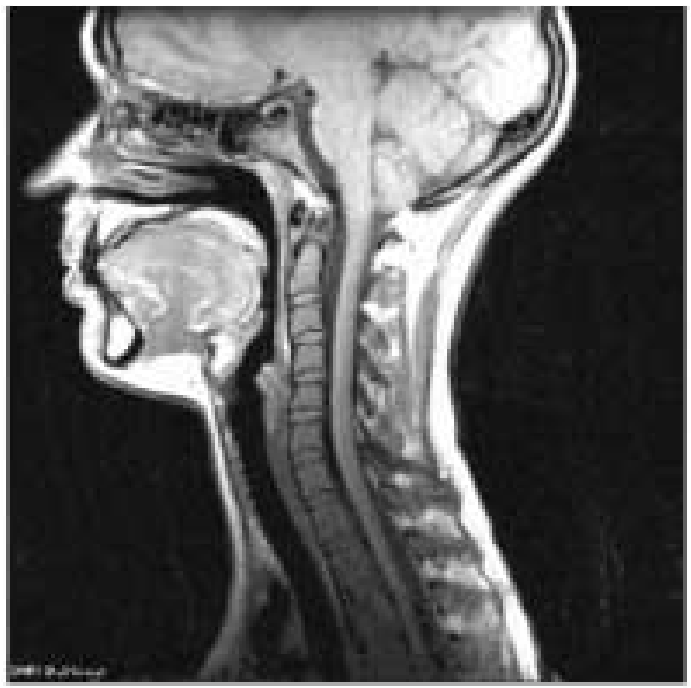

Figura 12. Detalhe de exame de ressonância magnética nuclear mostrando cabeça e pescoço de uma pessoa ( figura retirada do livro "The Basics of MRI" de J. P. Hornak[9]).

\section{Supercondutividade $\mathrm{MgB}_{2}$}

Desde a descoberta da supercondutividade a altas temperaturas em cerâmicas 15 , anos atrás, os pesquisadores praticamente ignoraram compostos metálicos simples porque, em geral, superconduzem a temperaturas muito baixas. Assim, foi um choque quando nos primeiros meses de 2001, a comunidade científica tomou conhecimento da descoberta de Akimitsu e seus colaboradores[10]. Um composto intermetálico com $\mathrm{T}_{c}=39 \mathrm{~K}, 16 \mathrm{~K}$ acima de qualquer outra liga metálica, tinha sido descoberto. Segundo Cava[11], o que torna a descoberta ainda mais fantástica é que ela foi feita praticamente ao acaso, por um grupo de cientistas que não estavam interessados em supercondutividade. Akimitsu e seu grupo estavam procurando um material semicondutor similar ao $\mathrm{CaB}_{6}$. Eles tentaram substituir o Ca por Mg, que está logo acima na tabela periódica. Como liga inicial, eles sintetizaram o $\mathrm{MgB}_{2}$, um composto simples com o processo de fabricação conhecido desde $1954[12,13]$ e vendido por fornecedores de materiais inorgânicos por aproximadamente 3 dólares o grama[14]. Tamanho deve ter sido o susto quando eles descobriram o valor da temperatura crítica do composto.

Segundo o artigo original de Akimitsu et al., as amostras de $\mathrm{MgB}_{2}$ foram preparadas na maneira usual, misturando-se Magnésio em pó (99.9\% Mg) e Boro amorfo, também em pó, (99\% B) na razão apropriada $(\mathrm{Mg}: \mathrm{B}=1: 2)$. As amostras foram, então, prensadas e aquecidas a $973 \mathrm{~K}$ sob alta pressão de argônio (196 $\mathrm{MPa}$ ), por 10 horas. Exames da amostra resultante por difração de raio $\mathrm{X}$ revelaram a formação da estrutura hexagonal do $\mathrm{MgB}_{2}$, como mostra a Fig. 13. O estado supercondutor foi, então, demonstrado, medindo a resistividade e a magnetização da amostra na presença de campo magnético. Na Fig. 14 podemos ver a susceptibilidade no $\mathrm{MgB}_{2}$. Devido ao efeito Meissner, a amostra se magnetiza na direção oposta ao campo magnético, e portanto a susceptibilidade, que é a razão da magnetização pelo campo magnético, é negativa. Quando o efeito Meissner não é perfeito, os valores da susceptibilidade ficam entre -1 e 0 , como é o caso. Na Fig. 15 podemos ver a perda da resistividade a $39 \mathrm{~K}$.
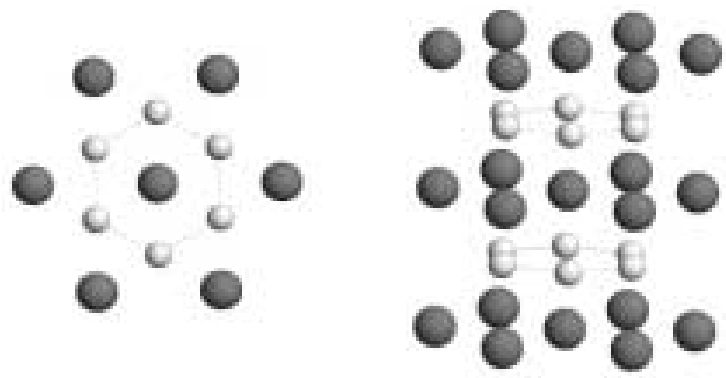

Figura 13. Estrutura cristalina do $\mathrm{MgB}_{2}$ mostrando as camadas hexagonais do $\mathrm{Mg}$ intercaladas com as camadas com estrutura de colméia de abelha do B (figura adaptada do artigo original de Akimitsu et al.[10])

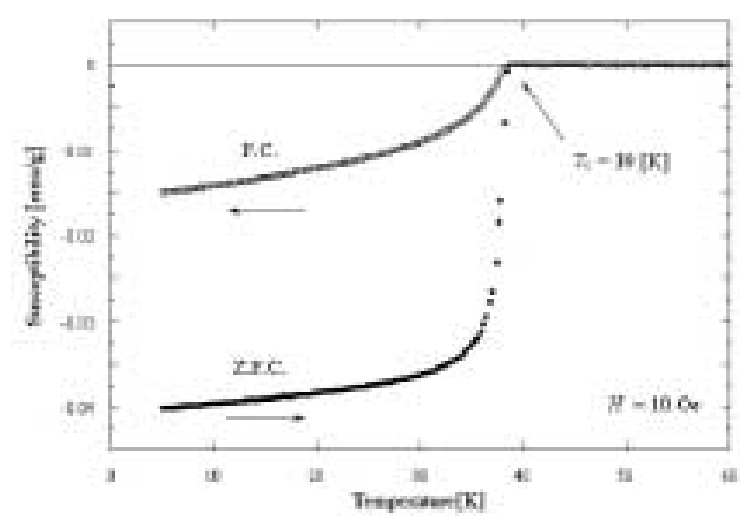

Figura 14. Susceptibilidade magnética $\chi$, que é a razão da magnetização pelo campo magnético aplicado, do $\mathrm{MgB}_{2}$ como função da temperatura obtidas utilizando duas técnicas diferentes. (figura retirada do artigo original de Akimitsu[10]). 


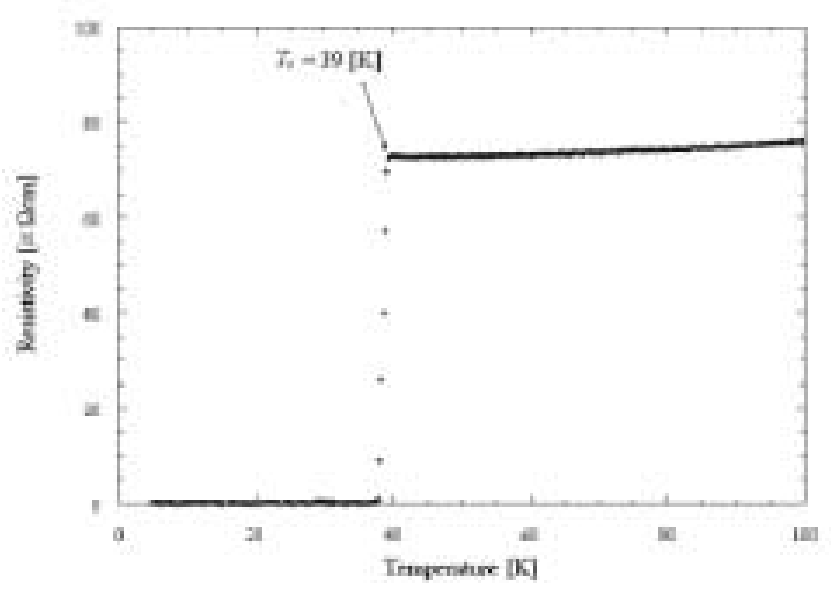

Figura 15. Resistividade do $\mathrm{MgB}_{2}$ como função da temperatura. (figura retirada do artigo original de Akimitsu[10]).

\section{A. Fabricação de $\mathrm{MgB}_{2}$ em pó e de- pendência isotópica de $\mathrm{T}_{c}$}

Logo após a divulgação da descoberta de Akimitsu et al., vários grupos ao redor do mundo começaram seus estudos para reproduzir e confirmar a descoberta dos japoneses. Entre eles, um grupo de cientistas do laboratório Ames, incluindo Paul Canfield, Doug Finnemore e Sergey Bud'ko[15, 16, 17], conseguiu sistematizar a produção de $\mathrm{MgB}_{2}$ em pó com alta pureza em um processo de duas horas. O processo consistia em misturar Mg (99.9\% puro) e B (99.5\%) na razão estequiométrica correta em um tubo de Ta, que era então selado em uma ampola de quartzo e colocado em um forno a $950^{\circ} \mathrm{C}$. Após duas horas, a ampola era retirada e o material resfriado à temperatura ambiente. Como o ponto de fusão do $\mathrm{Mg}$ é de $922 \mathrm{~K}$ e o do $\mathrm{B}$ é de $2573 \mathrm{~K}$, o processo de fabricação do $\mathrm{MgB}_{2}$ feito a $1222 \mathrm{~K}\left(950^{\circ} \mathrm{C}\right)$ acontece com $\mathrm{Mg}$ na fase líquida e $\mathrm{B}$ ainda na fase sólida. Isso, aliado ao fato de que quando pedaços grandes de B são utilizados a amostra resultante não é homogênea, levou os cientistas a perceberem que a reação se dá pela difusão dos átomos de $\mathrm{Mg}$ pelas partículas de B.

Após a sistematização da produção, eles fizeram substituições isotópicas. Trocando o isótopo ${ }^{11} \mathrm{~B}$, que forma $80 \%$ do Boro presente na natureza, por ${ }^{10} \mathrm{~B}$, eles descobriram que o $\mathrm{T}_{c}$ aumentava em $1.0 \mathrm{~K}$, veja Fig. 16. Essa dependência isotópica de $T_{c}$ verificada é consistente com a prevista na teoria BCS, ou seja, proporcional a $\mathrm{M}^{1 / 2}$. Assim, mesmo tendo um $\mathrm{T}_{c}$ incrivelmente grande, o $\mathrm{MgB}_{2}$ é um supercondutor convencional, com os fônons gerados pelos átomos de $\mathrm{B}$ mediando a interação entre elétrons na formação dos pares de Cooper.

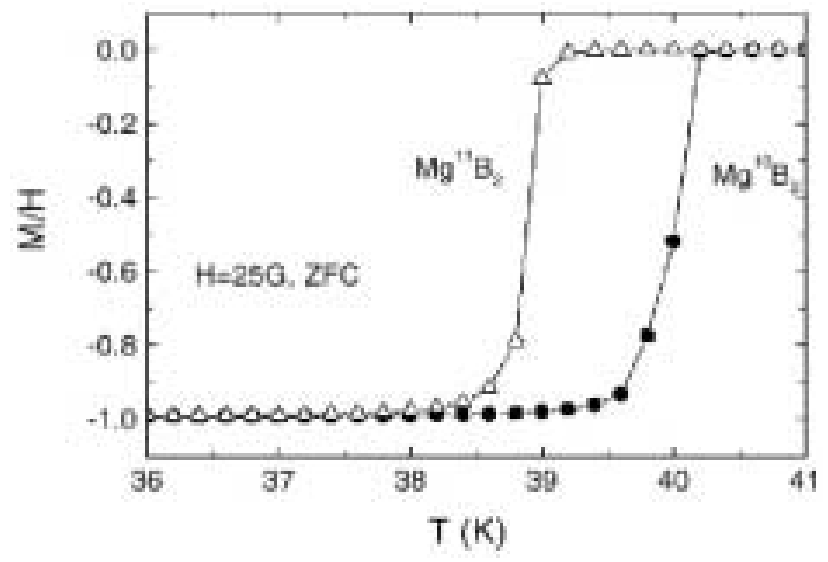

Figura 16. Susceptibilidade magnética $\chi$ do $\mathrm{MgB}_{2}$ como função da temperatura, mostrando a dependência isotópica de $\mathrm{T}_{c}$. (figura retirada do artigo de Canfield[17]).

\section{B. Fabricação de fios}

Dados o alto $\mathrm{T}_{c}$ do $\mathrm{MgB}_{2}$ e a abundância dos elementos $\mathrm{Mg}$ e $\mathrm{B}$ na crosta terrestre, (o magnésio é o oitavo elemento mais abundante na Terra), a questão imediata que fica é se o processo de fabricação de fios é simples e barato, e o principal, se os fios são capazes de transportar altas correntes elétricas. Este, de fato, é o problema principal enfrentado na utilização das cerâmicas supercondutoras em aplicações do dia a dia. Em um trabalho recente, Canfield[15] e colaboradores descrevem um processo de fabricação de fios bem simples, utilizando fibras de Boro e Magnésio fundido, veja Fig. 17. Como o ponto de fusão do $\mathrm{Mg}$ é de $922 \mathrm{~K}$ e o do B é de $2573 \mathrm{~K}$, o processo de fabricação dos fios feito a $1222 \mathrm{~K}\left(950^{\circ} \mathrm{C}\right)$ leva em conta a alta difusão do $\mathrm{Mg}$ pelas fibras de B. As fibras de B são seladas juntamente com Mg em pó, em um tubo de Ta, na razão estequiométrica correta, o tubo é, então, lacrado em uma ampola de quartzo e levado ao forno. Após aproximadamente duas horas de reação, a ampola é removida do forno e resfriada à temperatura ambiente. A aparência deformada dos fios, logo após a retirada do tubo de Ta, pode ser observada na Fig. 18. As fibras flexíveis e retas de B mostram-se deformadas e quebradiças após a reação. Segundo Canfield, os fios eram $80 \%$ densos e mostraram resistividade de $9.6 \mu \Omega \mathrm{cm}$ à temperatura ambiente. Isso quer dizer que, mesmo no estado normal, os fios de $\mathrm{MgB}_{2}$ são bons condutores de eletricidade, melhores até que o chumbo, cuja resistividade é $21 \mu \Omega \mathrm{cm}$. Os fios podiam ainda transportar correntes de até $\mathrm{J}_{c}=60 \mathrm{kA} / \mathrm{cm}^{2}$. O comprimento máximo alcançado foi de $5 \mathrm{~cm}$, mas fios maiores poderiam ser construídos, considerando-se a proteção externa com revestimento. 


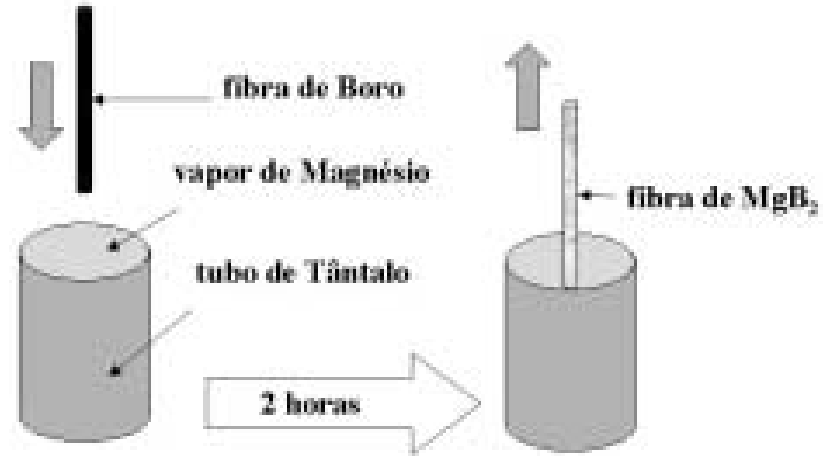

Figura 17. Processo de fabricação de fios supercondutores de $\mathrm{MgB}_{2}$, segundo Canfield[15].

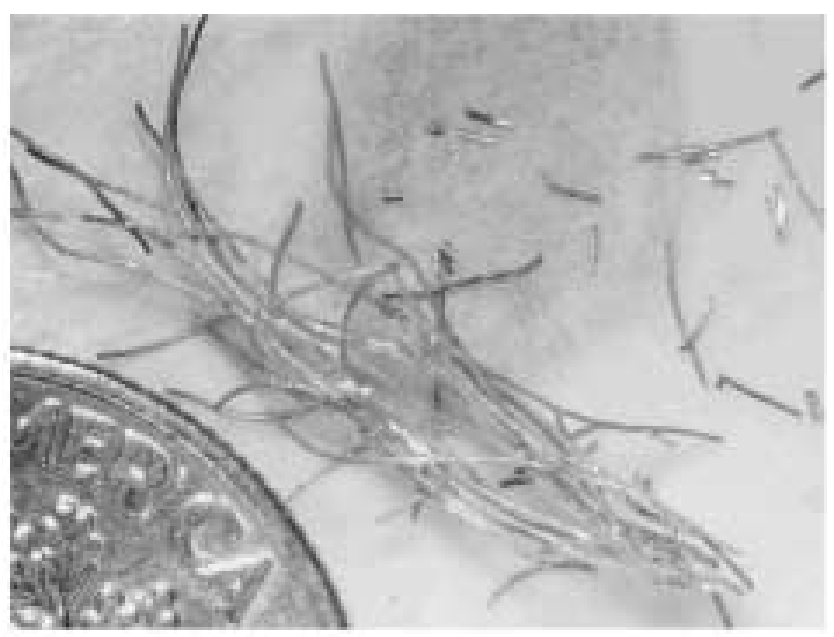

Figura 18. Fios de $\mathrm{MgB}_{2}$ depois de serem retirados do tubo de Ta e parte de uma moeda para escala. (figura retirada do artigo de Canfield[15]).

\section{Vantagens e aplicações em potencial}

$\mathrm{O}$ entusiasmo com a redescoberta do $\mathrm{MgB}_{2}$ justificase por duas razões principais. Primeiro, porque a liga, como vimos anteriormente, superconduz seguindo a teoria BCS[4]. Assim, diferentemente das cerâmicas supercondutoras, a liga parece ser um supercondutor convencional, como a maioria dos metais, mas com uma temperatura surpreendentemente alta. Segundo, porque, sendo uma liga metálica, é grande a expectativa de que ela se torne o material preferido na manufatura de fios que são a base para as aplicações do dia-a-dia. Com $\mathrm{T}_{c}$ de $39 \mathrm{~K}$, é bem possível que não seja necessária a refrigeração com hélio líquido, o que reduz significativamente os custos das aplicações.

\section{Conclusão}

Os estudos iniciais do $\mathrm{MgB}_{2}$ indicam que o material tem grande chance de se tornar o supercondutor preferido para aplicações, substituindo as até agora preferidas ligas de Nióbio. Assim, magnetos de alto campo, usados em máquinas de ressonância magnética, trens MAGLEVs, etc; cabos de transmissão; SMES e várias outras aplicações poderão ter seu custo reduzido com o uso do $\mathrm{MgB}_{2}$. Talvez em alguns anos um exame de ressonância magnética, por exemplo, saia pela metade do preço com o uso do $\mathrm{MgB}_{2}$ em vez das ligas de Nióbio.

Além das aplicações imediatas, a descoberta deste novo supercondutor intermetálico, com $\mathrm{T}_{c}$ tão alto, reacendeu a esperança na procura de um supercondutor à temperatura ambiente. Se tomarmos a tendência mostrada na Fig. 19, este sonho não parece estar tão distante. A descoberta da supercondutividade a $39 \mathrm{~K}$ no $\mathrm{MgB}_{2}$ é, então, mais uma esperança de que novos supercondutores intermetálicos com $\mathrm{T}_{c}$ recorde sejam descobertos.

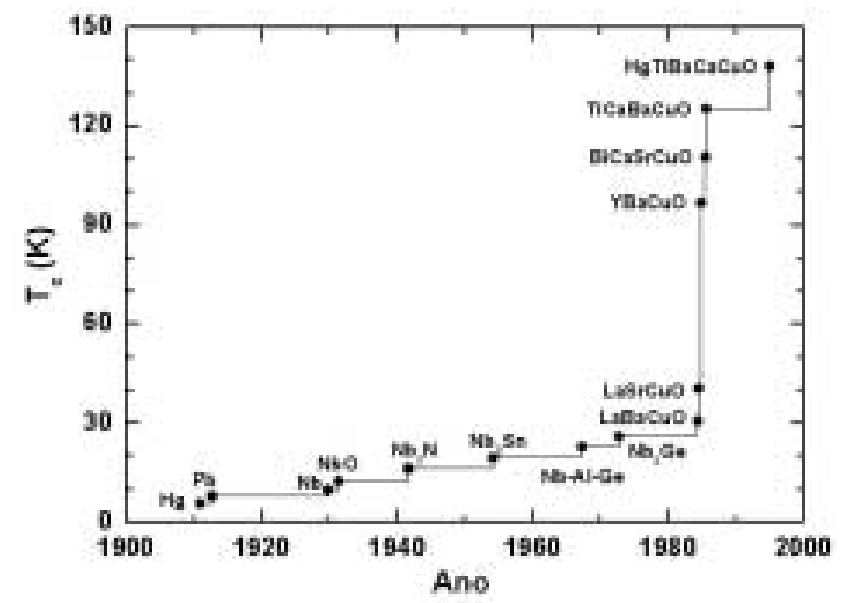

Figura 19. Temperatura de transição e o ano de descoberta (figura adaptada do livro "Superconductivity: its historical roots and development from mercury to the ceramic oxides" de P. F. Dahl[7], com a adição do supercondutor $\mathrm{Hg}_{0.8} \mathrm{Tl}_{0.2} \mathrm{Ba}_{2} \mathrm{Ca}_{2} \mathrm{Cu}_{3} \mathrm{O}_{8.33}$, que possui atualmente $\mathrm{o}$ $\left.\mathrm{T}_{c}[18]\right)$.

\section{References}

[1] H. K. Onnes, Commun. Phys. Lab. Univ. Leiden, Nos. 119, 120, 122 (1911).

[2] M. K. Wu, J. R. Ashburn, C. J. Torng, P. H. Hor, R. L. Meng, L. Gao, Z. J. Huang, Y. Q. Wang, C. W. Chu, Phys. Rev. Lett. 58(9), 908, (1987).

[3] Joe Eck. Superconductors.org. Disponível em: $<$ http://superconductors.org $>$. Acesso em: 8 de agosto de 2001.

[4] J. Bardeen, L. N.Cooper, J. R. Schrieffer, Phys. Rev. 108, 1175 (1957).

[5] F. Ostermann, L. M. Ferreira, C. J. H. Cavalcanti, Rev. Bras. Ens. Fís. 20, 270 (1998).

[6] J. G. Bednorz, K. A. Müller, Z. Phys. B 64, 189 (1986).

[7] P. F. Dahl, Superconductivity: its historical roots and development from mercury to the ceramic oxides, New York: American Institute of Physics, 1992, 406 p. 
[8] Maglev Systems

Development Department Home Page. Disponível em: <http://www.rtri.or.jp/rd/maglev/html/english/ maglev_frame_E.html $>$. Acesso em: 8 de agosto de 2001.

[9] J. P. Hornak, The Basics of MRI. Disponível em $<$ http://www.cis.rit.edu/htbooks/mri $>$. Acesso em 8 de agosto de 2001).

[10] J. Nagamatsu, N. Nakagawa, T. Muranaka, Y. Zenitani, J. Akimitsu, Nature 410, 63 (2001).

[11] R. J. Cava, Nature 410, 23 (2001).

[12] M. E. Jones, R. E. Marsh, J. Am. Chem. Soc. 76, 1434 (1954).

[13] R. M. Swift, D. White, J. Am. Chem. Soc. 79, 3641 (1957).
[14] CERAC Catalog Item Detail. Disponível em: $<$ http://asp.cerac.com/ProductDetail.asp?Item_No=M1006>. Acesso em 8 de agosto de 2001.

[15] P. C. Canfield, D. K. Finnemore, S. L. Bud'ko, J. E. Ostenson, G. Lapertot, C. E. Cunningham, C. Petrovic, Phys. Rev. Lett. 86, 2423 (2001).

[16] D. K. Finnemore, J. E. Ostenson, S. L. Bud'ko, G. Lapertot, P. C. Canfield, Phys. Rev. Lett. 86, 2440 (2001).

[17] S. L. Bud'ko, G. Lapertot, C. Petrovic, C. E. Cunningham, N. Anderson, P. C. Canfield, Phys. Rev. Lett. 86, 1877 (2001).

[18] P. Dai, B. C. Chakoumakos, G. F. Sun, K. W. Wong, Y. Xin, D. F. Lu, Physica C 243, 201 (1995). 